\title{
Hemodynamic Effects of Digoxin on Congestive Heart Failure in Old Myocardial Infarction, Dilated Cardiomyopathy, Acute Myocardial Infarction and Mitral Stenosis
}

\author{
Keiji Kurogane, M.D., Kazuhiro Fujtani, M.D., \\ and Hisashi Fukuzaki, M.D.
}

\section{Summary}

The hemodynamic effects of digoxin $(0.01 \mathrm{mg} / \mathrm{Kg})$ on congestive heart failure were compared in 32 patients with old myocardial infarction (OMI) $(n=9)$, dilated cardiomyopathy (DCM) $(n=10)$, acute myocardial infarction (AMI) $(n=5)$ and mitral stenosis (MS) $(\mathrm{n}=8)$.

The responses of heart rate (HR) and pulmonary capillary pressure (PCP) to digoxin in OMI, DGM and MS were marked but different in each of these groups and no significant changes were found in patients with AMI. The responses of cardiac index (CI) to digoxin in patients with OMI and DCM in whom left ventricular myocardial contractile force was impaired were divided into 2 groups (Group 1: CI increased more than 15\% and Group 2: less than 15\%). In Group 1, both CI and percent fractional shortening $(\% \mathrm{FS})$ before digoxin administration were lower than in Group 2, i.e., $1.97 \pm 0.27$ vs $2.80 \pm 0.48 \mathrm{~L} / \mathrm{min} / \mathrm{m}^{2} \quad(p<0.001)$ and $10.9 \pm 8.0$ vs $19.5 \pm 11.9 \%(\mathrm{p}<0.05)$, respectively. In MS, GI increased after digoxin administration only in the 2 patients with low CI and rapid HR in the control state.

These results indicate that the mode of hemodynamic response to digoxin is considerably different in various diseases. They further suggest that digoxin should not be used in the early phase of AMI, although digoxin was of great clinical benefit in patients with OMI and DCM through such mechanisms as its positive inotropic and negative chronotropic effects and lowering of PCP.

\section{Additional Indexing Words :}

Digoxin Hemodynamics Congestive heart failure

From the Department of Internal Medicine, Division 1, Kobe University School of Medicine, Kusunoki-cho, Kobe 650, Japan.

Received for publication March 2, 1984. 
D IGITALIS glycosides have been used in the therapy of congestive heart failure for the more than 200 years since the report of Withering and continue to be recognized as extremely useful drugs. The postulated mechanism of the hemodynamic effects of digitalis on congestive heart failure is still based upon study results obtained more than 10 years ago. These studies involved acute observations from 30 to $120 \mathrm{~min}$ after intravenous administration of digitalis. However, there are some problems that remain unsolved, e.g., whether digitalis is effective in acute myocardial infarction and mitral stenosis and whether digitalis can necessarily increase cardiac index in congestive heart failure. ${ }^{1)}$

The hemodynamic responses are now easily observed over a longer period of time using a Swan-Ganz thermodilution catheter. However, except in cases of acute myocardial infarction and circulatory shock, ${ }^{21-6)}$ there have been few reports on the hemodynamic effects of digitalis in recent years. Marked progress has been made in the drug treatment of congestive heart failure since diuretics and vasodilator drugs have been employed. Accordingly, the indication for the use of digitalis in the treatment of congestive heart failure is now more strictly defined.

To clarify these problems and to decide on appropriate indications for the use of digitalis, we compared the hemodynamic responses to digoxin of patients with congestive heart failure secondary to old myocardial infarction, dilated cardiomyopathy, acute myocardial infarction and mitral stenosis.

\section{Methods}

Thirty-two patients with congestive heart failure of NYHA functional classes II-IV were studied. There were 23 men and 9 women with a mean age of 55 years, ranging from 27 to 82 years. The etiology of congestive heart failure was either old myocardial infarction (OMI) $(n=9)$, dilated cardiomyopathy (DCM) $(n=10)$, acute myocardial infarction (AMI) $(n=5)$ or mitral stenosis (MS) $(n=8)$. Myocardial infarction was documented by a history of characteristic chest pain, the development of pathologic $Q$ waves on the ECG and an increase in creatine phosphokinase. AMI and OMI were defined as those cases where the period from the onset of attack was within 3 days and 1 month or more, respectively. DCM was diagnosed on the basis of its typical clinical course in the absence of any other heart diseases based upon echocardiography, coronary arteriography and myocardial biopsy. The diagnosis of MS was confirmed by echocardiography and no MS patient had any other valvular disease. Atrial fibrillation was present in a DCM case and all MS cases. None of the 32 patients had been given digitalis 
before. The administration of diuretics and vasodilator drugs was stopped for 24 hours before the study.

Hemodynamic measurements: Before the study, echocardiographic parameters such as left ventricular end-diastolic dimension (LVDd), left ventricular end-systolic dimension (LVDs) and fractional shortening (\%FS) were measured. After informed consent had been obtained, a Swan-Ganz triple lumen thermodilution catheter was inserted via the cubital vein and its tip was positioned in the pulmonary artery. Cardiac output was measured by computer model 9520 and determined in triplicate. Heart rate (HR) was measured by EGG and blood pressure (BP) by sphygmomanometer. Pulmonary capillary pressure (PCP) and right atrial pressure (RAP) were measured with

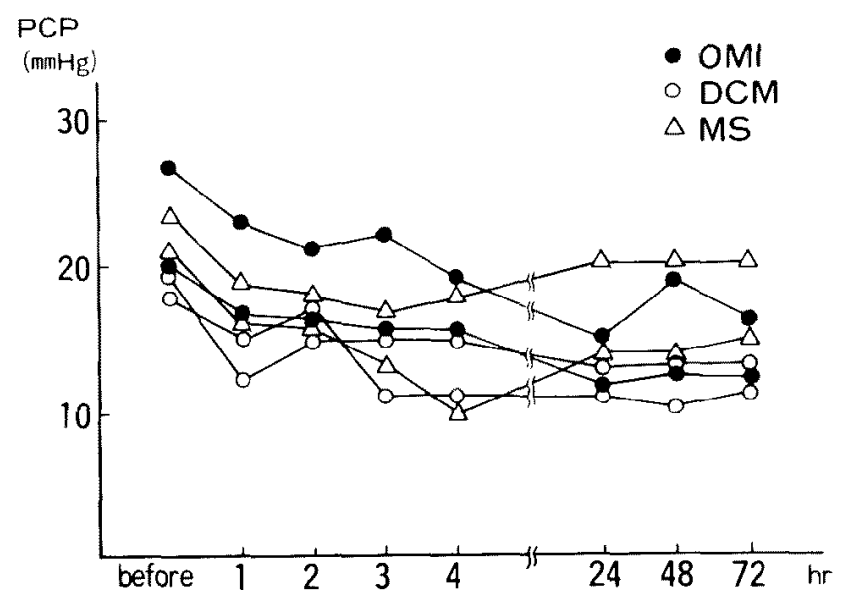

Fig. 1. Response of pulmonary capillary pressure to digoxin in 6 patients.
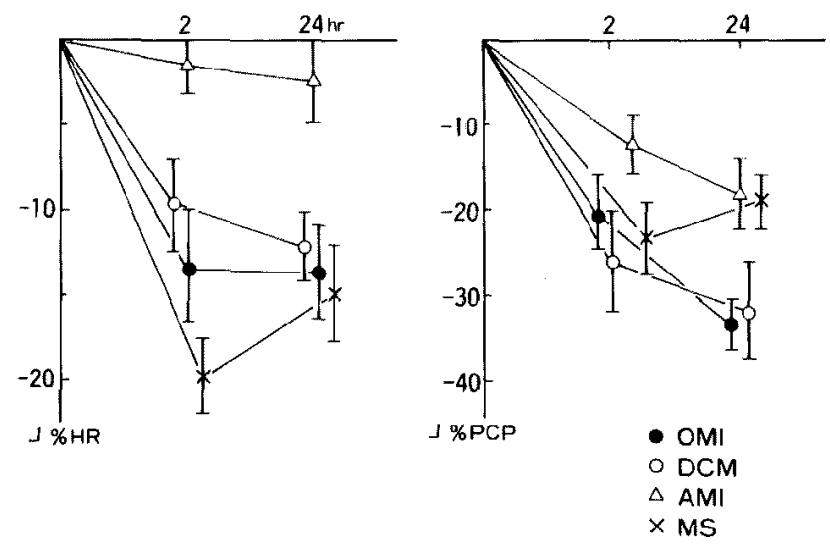

Fig. 2. \% change of heart rate and pulmonary capillary pressure by digoxin administration. 
Table I. Hemodynamic Responses to Intravenous Digoxin in (OMI), Dilated Gardiomyopathy (DGM), Acute My-

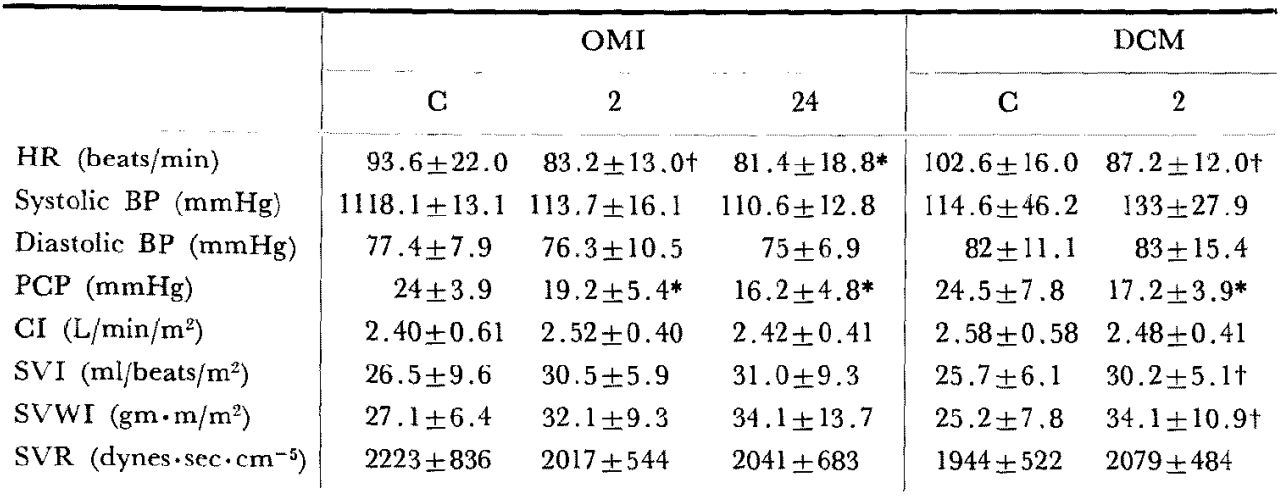

Values are expressed as mean $\pm \mathrm{SD}$.

Abbreviations : $\mathrm{C}=$ control ; $\mathrm{HR}=$ heart rate; $\mathrm{BP}=$ blood pressure ; $\mathrm{PCP}=$ pulmonary capillary pressure; $\mathrm{CI}=$ cardiac index; $\mathrm{SVI}=$ stroke volume index; SVWI=stroke volume work index;

a Siemens 746 transducer and recorded on a Siemens mingograph. Hemodynamic values were calculated using standard formulas. Mean BP (mBP) was calculated from the formula $1 / 3(\mathrm{sBP}-\mathrm{dBP})+\mathrm{dBP} \mathrm{mmHg}$, stroke volume index (SVI) was $\mathrm{CI} / \mathrm{HR} \mathrm{ml} / \mathrm{m}^{2}$, stroke volume work index (SVWI) was calculated from the formula $(\mathrm{sBP}-\mathrm{PCP}) \times \mathrm{SI} \times 1.36 / 100 \mathrm{gm} \cdot \mathrm{m} / \mathrm{m}^{2}$. Systemic vascular resistance (SVR) was derived from the formula $\mathrm{BP} \times 80 / \mathrm{CI}$ dynes $\cdot \mathrm{sec} \cdot \mathrm{cm}^{-5}$.

Protocol: After control hemodynamic measurements were obtained, digoxin $0.01 \mathrm{mg} / \mathrm{Kg}$ was administered intravenously for $10 \mathrm{~min}$ and hemodynamic measurements were carried out at 1, 2, 4 and 24 hours after the administration. Thereafter, digoxin $0.05 \mathrm{mg} / \mathrm{Kg}$ p.o. was administered every 24 hours. In 6 patients ( 2 with OMI, 2 with DGM and 2 with MS), further measurements were made 48 and 72 hours after drug administration.

Statistical analysis was performed using Student's paired t-test.

\section{Results}

As a preliminary study, we observed the hemodynamic effects of digoxin in 6 patients (2 with OMI, 2 with DCM, 2 with MS) for 72 hours after its administration. PCP was lowered during the first 24 hours after drug administration but thereafter no significant change was found and the response in patients with MS was different from that in other diseases during the first 24 hours (Fig. 1). Based upon these results, we compared the hemodynamic changes in patients with various diseases associated with congestive heart failure in the 24 hour period after digoxin administration. 
Congestive Heart Failure Due to Old Myocardial Infarction ocardial Infarction (AMI) and Mitral Stenosis (MS)

\begin{tabular}{|c|c|c|c|c|c|c|}
\hline & \multicolumn{3}{|c|}{ AMI } & \multicolumn{3}{|c|}{ MS } \\
\hline 24 & $\mathrm{C}$ & 2 & 24 & $\mathrm{C}$ & 2 & 24 \\
\hline $88.5 \pm 12.7^{*}$ & $113.4 \pm 17.1$ & $111.8 \pm 15.4$ & $111.3 \pm 17.2$ & $99.9 \pm 24.7$ & $80.4 \pm 21.0^{*}$ & $84.3 \pm 19.0^{*}$ \\
\hline $126.1 \pm 23.6$ & $120.5 \pm 9.3$ & $121.5 \pm 10.0$ & $120 \pm 18.8$ & $123.5 \pm 21.9$ & $120.8 \pm 17.4$ & $114.8 \pm 18.1$ \\
\hline $79.8 \pm 9.8$ & $89.2 \pm 7.2$ & $88 \pm 10.3$ & $85.2 \pm 13.2$ & $80.5+7.7$ & $79.1 \pm 8.0$ & $74.3 \pm 9.8$ \\
\hline $16.1 \pm 4.9^{*}$ & $20.6 \pm 5.2$ & $18 \pm 6.4$ & $17 \pm 5.6$ & $20.9 \pm 4.5$ & $16.5 \pm 5.6^{*}$ & $16.6 \pm 4.8^{*}$ \\
\hline $2.57 \pm 0.48$ & $2.90 \pm 1.06$ & $2.96 \pm 1.06$ & $2.81 \pm 1.08$ & $2.25 \pm 0.40$ & $2.23 \pm 0.37$ & $2.38 \pm 0.26$ \\
\hline $29.3 \pm 5.5 t$ & $25.7 \pm 9.2$ & $26.7 \pm 9.0$ & $25.4 \pm 9.4$ & $24.0 \pm 8.9$ & $29.2 \pm 11.8 \dagger$ & $28.7 \pm 6.9 \dagger$ \\
\hline $32.3 \pm 6.6^{*}$ & $28.1 \pm 6.4$ & $30.4 \pm 5.4$ & $27.4 \pm 5.6$ & $25.4 \pm 10.8$ & $31.6 \pm 14.9+$ & $29.2 \pm 9.1 \dagger$ \\
\hline $1985 \pm 523$ & $1872 \pm 700$ & $1823 \pm 631$ & $1872 \pm 704$ & $2338 \pm 532$ & $2271 \pm 397$ & $1945 \pm 202$ \\
\hline
\end{tabular}

Hemodynamic responses to digoxin in each diseasc group are shown in Table I and Fig. 2. HR (beats/min) significantly decreased at 2 hours from $93.6 \pm 22.0$ to $83.2 \pm 13.0$ in OMI, from $102.6 \pm 16.0$ to $87.2 \pm 12.0$ in DCM and from $99.9 \pm 24.7$ to $80.4 \pm 21.0$ in MS and a further reduction was found at 24 hours to $81.4 \pm 18.8$ in OMI and to $88.5 \pm 12.7$ in DCM, but in MS, on the contrary, a slight elevation to $84.3 \pm 19.0$ was found.

There were no significant changes in systolic and diastolic blood pressure in these disease groups. PCP $(\mathrm{mmHg})$ was decreased significantly at 2 hours from $24 \pm 3.9$ to $19.2 \pm 5.4$ in $\mathrm{OMI}$, from $24.5 \pm 7.8$ to $17.2 \pm 3.9$ in DCM and from $20.9 \pm 4.5$ to $16.5 \pm 5.6$ in $\mathrm{MS}$ and at 24 hours it further decreased to $16.2 \pm 4.8$ in OMI, and to $16.1 \pm 4.9$ in DCM, but no further reduction was found in MS. In terms of these parameters, no significant change was demonstrated in AMI at either 2 or 24 hours after digoxin administration. CI was not significantly increased in any disease group at either 2 or 24 hours. SVI and SVWI were significantly increased in DCM and MS at both periods although no significant change was found in OMI and AMI. No significant change in SVR was found in any disease group at either period.

According to the summarized data, no significant change in CI was demonstrated as mentioned above. However, when looking at individual cases, it was noted that there were cases with and other without digoxin induced changes in CI. Therefore, patients with OMI and DCM, where the mechanism of heart failure is due to impaired left ventricular contractile force were divided into 2 groups. Group 1 consisted of 7 patients in whom CI increased by $15 \%$ or more at 2 hours after digoxin administration and Group 2 consisted of 12 patients in whom CI increased by less than $15 \%$ at the same 
Table II. Comparison of Clinical Features, Hemodynamic Parameters and Echocardiographic Parameters in Responders (Group 1) and Non-responders (Group 2) to Intravenous Digoxin

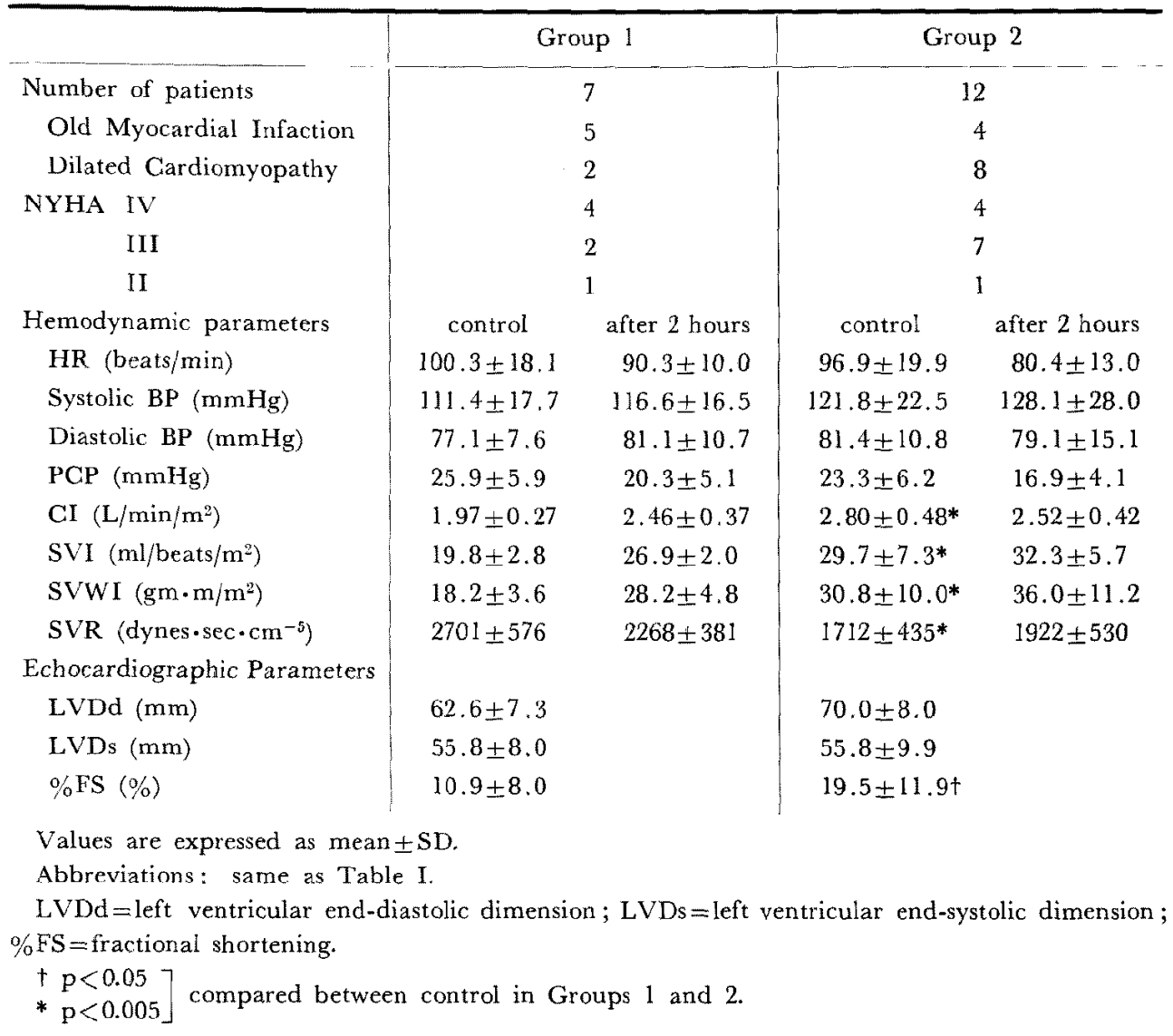

period. In these 2 groups, clinical features and hemodynamic and echocardiographic parameters were compared (Table II).

CI before digoxin administration was lower in Group 1 than that in Group $2\left(1.97 \pm 0.27\right.$ vs $\left.2.80 \pm 0.48 \mathrm{~L} / \mathrm{min} / \mathrm{m}^{2}, \mathrm{p}<0.05\right)$. After digoxin administration CI increased to $2.46 \pm 0.48 \mathrm{~L} / \mathrm{min} / \mathrm{m}^{2}$ in Group 1, while it decreased to $2.52 \pm 0.42 \mathrm{~L} / \mathrm{min} / \mathrm{m}^{2}$ in Group 2. PCP, on the other hand, decreased to nearly the same level in both groups (Fig. 3). SVI, SVWI and $\% \mathrm{FS}$ before digoxin administration in Group 1 were significantly lower than those in Group 2, while SVR in Group 1 was higher than that in Group 2 $(p<0.001)$. In other hemodynamic and echocardiographic parameters, no significant difference was found between the 2 groups. The severity of heart failure expressed by NYHA functional classification was greater in Group 1 than in Group 2. In MS, CI increased after digoxin administration in only 2 cases whose HR were markedly increased and CI were markedly decreased 


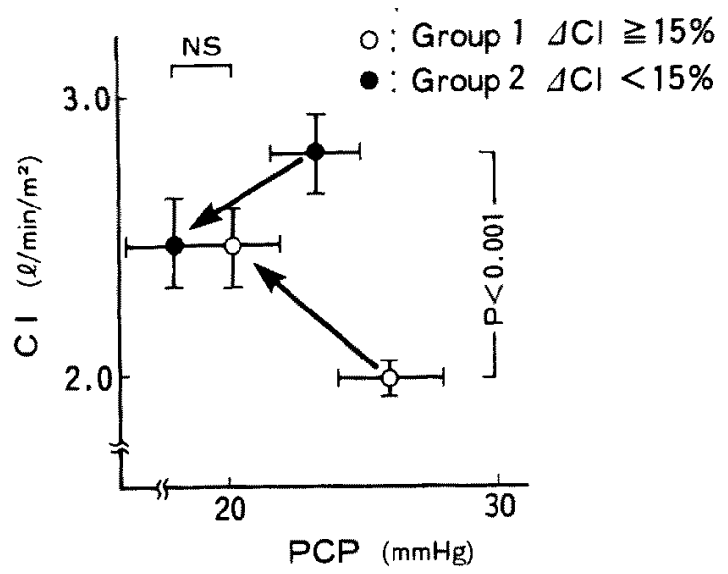

Fig. 3. Response of cardiac index and pulmonary capillary pressure to digoxin in patients with old myocardial infarction and dilated cardiomyopathy.

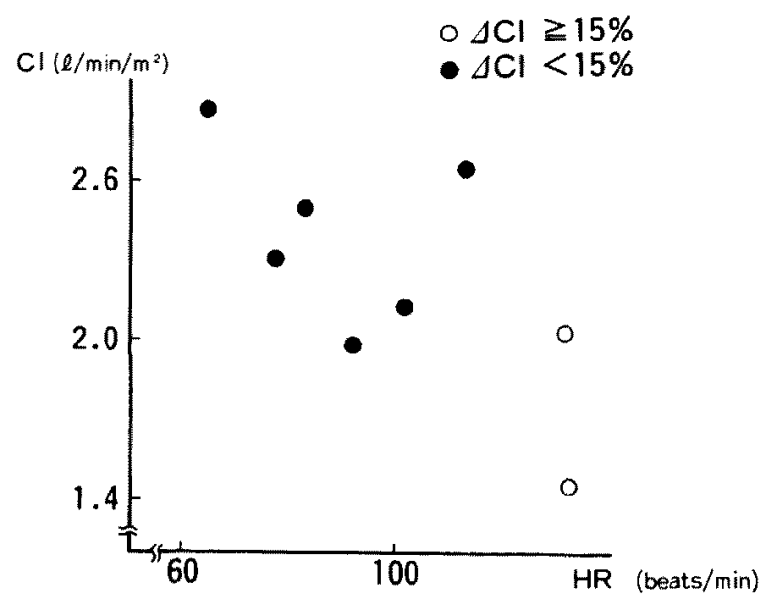

Fig. 4. Response of cardiac index and heart rate to digoxin in patients with mitral stenosis.

at baseline (Fig. 4).

The serum digoxin level was determined by radioimmunoassay in all patients after 24 hours of digoxin administration and was in the therapeutic range with levels of between 0.22 and $1.46 \mathrm{ng} / \mathrm{ml}$ (mean, $0.72 \pm 0.35 \mathrm{ng} / \mathrm{ml}$ ).

\section{Discussion}

In AMI, no significant changes in hemodynamic parameters were revealed after digoxin administration. Three mechanisms to explain the lack of responsiveness to digoxin have been proposed as follows: (1) Tennant and 
Wiggers implicated the paradoxical wall motion in the infarcted area." Langley et al, more recently, pointed out the considerable amount of "wasted work" due to the paradoxical wall motion. ${ }^{8)}$ Digitalis may augment the contractility of the non-infarcted myocardium but such effect is cancelled out by the paradoxical wall motion of the infarcted area. (2) Circulating catecholamine is increased in the early stage of acute myocardial infarction. ${ }^{9), 10)}$ This causes an increase in the contractility of the non-infarcted myocardium and in such a situation further augmentation of contractility by digoxin may not be expected. (3) Myocardial depressant factor (MDF) may play an important role in supressing the myocardial contractility, ${ }^{111}$ whereby the action of digoxin may be equivocal. Since such a mechanism, if present, persists very briefly during the early phase of AMI, digoxin is considered to be effective several days after the onset of AMI. In addition, as other reports have suggested that digitalis augments serum CPK and increases mortality in patients with $A M I,{ }^{121,13)}$ it is advisable that digoxin not be used during the early phase of AMI.

The effect of digoxin on HR was most prominent in MS compared with other diseases, although its effect on PCP was small. An increase in CI was found in only 2 MS patients with rapid $\mathrm{HR}$ and low CI at baseline. In these 2 cases, digoxin resulted in the prolongation of diastole and thus augmented left ventricular filling volume and CI. In the other 6 MS cases whose CI did not increase, HR and PCP were reduced slightly. All MS cases exhibited atrial fibrillation. Previous reports have presented little evidence of a beneficial effect of digitalis in pure MS patients with normal sinus rhythm and it was suggested that digitalis might be harmful to such patients. ${ }^{14)}$ We observed the hemodynamic effects of digoxin according to the same protocol in 2 cases with pure MS and normal sinus rhythm who were in NYHA class I with normal CI and demonstrated that digoxin did not increase CI but decreased PCP and HR similar to the response in patients with atrial fibrillation.

The most significant improvement of various hemodynamic parameters was found in patients with DCM and OMI, in whom left ventricular contractile force was decreased, but CI was not necessarily increased. These results are in agreement with several other reports. Data reviewed by Selzer and Malmborg from all studies before 1962 revealed that in two thirds of 132 patients, digoxin increased CI but in the remaining one third, no beneficial effect was demonstrated. ${ }^{15}$ ) They could not find any difference in the response to digoxin between the 2 groups, i.e., the group with increased CI and the group without increased CI. We also compared the hemodynamic parameters and clinical features of these 2 groups. CI and \% FS before digoxin 
administration in the former group was significantly lower than those in the latter group. Therefore, the improvement of left ventricular function due to digoxin administration in the former group was probably caused by the enhancement of myocardial contractility which is a well-known cardiac effect of digoxin. In the latter group, on the other hand, digoxin reduced PGP without increasing CI. Regarding its mechanism in this group, it was suggested that the vasoconstrictive effect of digoxin on veins, especially the hepatic vein, induced blood pooling in the portal venous system, which resulted in diminished venous return. ${ }^{16}$ ) We could not differentiate between groups by their clinical features. However, in all patients in NYHA class IV (4 pts in each group), digoxin administration improved symptoms such as orthopnea and nocturnal dyspnea. Therefore, it was postulated that digoxin improved the clinical features as a result of its ability to lower PCP and an increase in CI was not necessarily needed.

In summary, we concluded the following.

1. Clinical use of digoxin is not advisable in the early phase of AMI.

2. In MS, digoxin reduced $\mathrm{HR}$ and PGP through its negative chronotropic effect and its effect was more pronounced in cases with markedly increased HR.

3. In DCM and OMI, digoxin increased CI only in cases with markedly lowered $\mathrm{CI}$ and \% FS, but reduced $\mathrm{PCP}$ in all cases.

\section{REFERENCES}

1. Cohn K, Selzer A, Kersh ES: Variability of hemodynamic response to acute digitalization in chronic cardiac failure due to cardiomyopathy and coronary artery disease. Am J Cardiol 35: 461,1975

2. Hodges M, Friesinger GC, Riggins RCK: Effect of intravenously administered digoxin on mild left ventricular failure in acute myocardial infarction in man. Am J Cardiol 29: 749, 1972

3. Balcon $\mathrm{R}$, Hoy J, Sowton $\mathrm{E}$ : Hemodynamic effects of rapid digitalization following acute myocardial infarction. Br Heart J 30: 373, 1968

4. Cohn JN, Tristani FE, Khatri IM: Cardiac and peripheral vascular effects of digitalis in clinical cardiogenic shock. Am Heart J 78: 318, 1969

5. Karliner JS, Braunwald E: Present status of digitalis treatment of acute myocardial infarction. Circulation 45: 891, 1972

6. Lipp $\mathrm{H}$, Denes $\mathrm{P}$, Resnekov L: Hemodynamic response to acute intravenous digoxin in patients with recent myocardial infarction and coronary insufficiency with and without heart failure. Chest 63: 862, 1973

7. Tennant $R$, Wiggers GJ: The effect of coronary occlusion on myocardial contraction. Am J Physiol 112: 351, 1935

8. Langley JO, Martinez A, Fakhro A: Paradoxical precordial motion and wasted left ventricular work: the concept of cardiac dyssynergy. Am Heart J 73: 349, 1967

9. Beiser GD, Epstein SE, Goldstein RE: Comparison of the peak inotropic effects of a catecholamine and a digitalis glycoside in the intact canine heart. Circulation 42: 805, 1970 
10. Goldstein RA, Passamani ER, Roberts R: A comparison of digoxin and dobutamine in patients with acute infarction and cardiac failure. New Engl J Med 303: 846, 1980

11. Henderson AH, Most AS, Sonnenblick EH: Depression of myocardial contractility in rats by free fatty acids during hypoxia. Circ Res 26: 439, 1970

12. Varonkov Y, Shell WE, Smirnov V: Augmentation of SERUM CPK activity by digitalis in patients with acute myocardial infarction. Circulation 55: 719, 1977

13. Moss AJ, Davis HT, Cohard DL: Digitalis-associated cardiac mortality after myocardial infarction. Circulation 64: 1150, 1981

14. Akbarian M, Yankopoulos NA: Hemodynamic effects of ouabain in patients with mitral stenosis and sinus rhythm. Am J Cardiol 21: 241, 1968

15. Selzer A, Malmborg RO: Hemodynamic effects of digoxin in latent cardiac failure. Circulation 25: 695, 1962

16. Ross J Jr, Braunwald E, Waldhausen JA: Studies on digitalis. II. Extra cardiac effects on venous return and on the capacity of the peripheral vascular bed. J Clin Invest 29: 937, 1960 